\title{
Jeux sportifs collectifs et handicap Genèse de pratiques partagées innovantes $^{1}$
}

\author{
Jean-Pierre GAREL \\ Chercheur associé au Laboratoire Cultures-Éducation-Sociétés (LACES EA 4140) \\ Équipe ERCEP3, Université Bordeaux Segalen
}

Résumé: La participation de personnes en situation de handicap et de personnes ordinaires à un même jeu sportif collectif ne va pas de soi. Au-delà de raisons qui conduisent à concevoir et développer des pratiques partagées, notamment lors d'un tel jeu, et de l'identification des obstacles qui entravent la réussite de ce partage, il s'agit d'étudier des jeux sportifs collectifs qui se démarquent de l'uniformité des règles et de la logique sportive dominante pour créer des conditions de pratique plus équitables. Trois exemples de ces jeux innovants sont considérés: le jeu de thèque adapté aux enfants aveugles, le basketball adapté aux personnes déficientes visuelles, et le baskin, conçu pour des personnes ayant tout type de déficience. Ils se distinguent par leur degré de codification et d'institutionnalisation. Sont présentés leur architecture et leur développement, afin de s'interroger sur leur pertinence et d'identifier les éléments favorables à la réussite de ces innovations.

Mots-clés: Déficience visuelle - Équité - Handicap - Inclusion - Innovation - Jeu collectif - Participation sociale - Pratique partagée.

\section{Collective sport games with disability. Genesis of innovative shared practices}

Summary: The participation of persons who are in a situation of disability and mainstream persons in the same collective sporting game is not a simple question. This article start by discussing reasons leading to the design and the development of shared practices, in particular in such a game, and obstacles hindering the success of this sharing. Mainly, it studies collective sports games that deviate from the dominant uniformity of sports rules and logic with the aim of creating more equitable conditions of sports practice. Three examples of these sports innovative activities, distinguished by their degree of codification and institutionalization, are examined: thèque game adapted to blind children, basketball adapted to visually impaired people, and baskin, designed for people with any type of disability. Their architecture and development are presented, in order to question their relevance and the elements of succes of these innovations.

Keywords: Collective game - Disability - Equity - Inclusion - Innovation - Shared practice - Social participation Visual impairment.

\footnotetext{
1. Cet article reprend partiellement, avec le même titre, un article extrait du dossier "Pratiques sportives et handicap: de la transformation à la mise en scène des corps différents ", dirigé par E. Lantz et A. Marcellini et publié par la Revue Développement humain, handicap et changement social - Journal of Human Development, Disability, and Social Change, 23(1), 7-24. RIPPH (Réseau international sur le processus de production du handicap), septembre 2017.
} 
Les jeux sportifs occupent une place particulière au sein des activités physiques, sportives et artistiques. Contrairement à d'autres, comme les activités de pleine nature (escalade, voile...) ou à caractère artistique (danse, arts du cirque...), ils impliquent un affrontement codifié (Parlebas, 1981). Dans ces conditions, en milieu ordinaire, les personnes avec une déficience risquent de ne pas être à la hauteur des autres joueurs et de ne pas trouver dans ces activités un vecteur d'inclusion bien adapté. Pour qu'elles puissent malgré tout y participer avec plaisir et profit, une prise en compte des singularités s'impose, avec des obstacles à surmonter, en particulier une logique sportive qui s'accommode mal de rencontres entre des compétiteurs aux capacités et performances très dissemblables.

La réflexion sur les pratiques partagées par des individus aux capacités jugées a priori incompatibles est traversée par des questions sur l'égalité et l'équité, et aussi, de façon plus pragmatique, par des questions d'ordre didactique qui sont au cœur d'une problématique très vive chez les enseignants: la gestion de l'hétérogénéité des élèves.

Au-delà d'un bref exposé de raisons qui conduisent à développer des pratiques partagées, en particulier lors de jeux sportifs collectifs, et de l'identification d'obstacles qui entravent la réussite de ce partage, nous étudierons des jeux sportifs collectifs qui se démarquent de l'uniformité des règles, et donc de la logique sportive dominante, pour créer des conditions de pratique plus équitables. Trois exemples de ces jeux seront considérés: le jeu de thèque adapté aux enfants aveugles, le basketball adapté aux personnes déficientes visuelles, c'est-à-dire aveugles ou malvoyantes, et le baskin, conçu pour des personnes ayant tout type de déficience.

\section{PROBLÉMATIOUUE}

II est ici question de souligner l'intérêt d'une étude portant sur des jeux sportifs collectifs partagés, en mentionnant un enjeu fondamental dont ils sont porteurs, d'évoquer des obstacles auxquels se heurte leur pratique, de montrer l'originalité du thème abordé, à partir d'une revue de littérature, et de préciser notre questionnement.

\section{Des pratiques à fort enjeu social}

Prud'homme et Goupil (2001) ont montré que les jeux partagés peuvent participer à la construction de compétences sociales. Elles se sont penchées sur des jeux regroupant des enfants d'âge préscolaire et scolaire, les uns avec une déficience, les autres sans. À partir d'une revue de littérature sur les groupes de jeu intégré, composés, d'une part, d'enfants ayant une déficience intellectuelle ou présentant un trouble envahissant du développement, tel l'autisme, et d'autre part de pairs sans déficience, elles ont mis en évidence les avantages de l'intégration, par rapport au milieu spécialisé, pour l'apprentissage et l'évolution des habiletés ludiques et sociales. Pour ce qui est des jeux sportifs collectifs, ils ouvrent sur un champ de participation sociale dont un aspect fondamental tient à la possibilité de jouer des " rôles sociaux qui sont valorisés par la personne ou par son contexte socioculturel " (Fougeyrollas, 2009, p. 169), et donc à l'exercice des droits individuels dans la vie de tous les jours. Or, I'enfant ou l'adolescent exclu des activités "ludomotrices » (Parlebas, op. cit, 


\section{DOSSIER}

p. 139) ne peut pas exercer comme les autres son droit d'écolier, de collégien ou de lycéen à suivre l'ensemble des enseignements. II subit aussi une restriction de participation sociale quand, pour ses loisirs, il aspire en vain à pratiquer des activités qui appartiennent au patrimoine culturel enfantin ou qui sont l'expression d'une culture sportive médiatisée. En être exclu contrarie le désir de se retrouver entre camarades en milieu ordinaire et de satisfaire un besoin de sociabilité particulièrement vif à l'adolescence.

\section{Des obstacles à une pratique partagée}

Selon une enquête conduite par la commission Études et recherches de la Fédération française du sport adapté (Bui-Xuân et Mikulovic, 2006), la compétition paraît exclure les pratiquants les moins performants. En effet, parmi les personnes présentant une déficience intellectuelle, ce sont celles dont les troubles cognitifs sont les plus légers qui sont majoritairement présentes dans les associations sportives ordinaires. Ce fait est corroboré et assorti d'une explication par une enquête menée sur le terrain: le niveau de performance attendu pour être engagé dans une compétition rejette celles et ceux qui ne s'en montrent pas capables (Gouat, 1999).

À l'école ordinaire, les élèves en situation de handicap ne sont pas toujours inclus dans les jeux sportifs collectifs. C'est ce que l'on voit dans un collège où les adolescents ayant une déficience intellectuelle ne participent pas au cycle de basketball avec les autres élèves (Garel, 2001). Selon leurs enseignants, ils ont trop de difficultés pour y trouver une réussite suffisamment gratifiante.

La difficulté d'inclusion dans les jeux sportifs collectifs est accentuée lorsque la compétition est vécue par un joueur comme étant lourde d'enjeu, mettant sur lui une pression qu'il supporte d'autant moins qu'il est peu performant et qu'une situation de handicap peut s'accompagner d'une confiance en soi altérée et de vulnérabilité (Gardou, 2005).

Par ailleurs, parce qu'elle est normalisatrice, I'organisation dominante du sport n'est pas favorable à des rencontres entre pratiquants très différents. La rencontre sportive est généralement fondée sur l'homogénéité des concurrents, de façon à préserver autant que possible l'égalité des chances dans la compétition. C'est dans ce sens qu'est conçue la structure pyramidale du football en France, selon un système de classement officiel des ligues et des divisions rassemblant chacune des équipes de niveau comparable. Cette homogénéité conduit à se demander si la logique sportive ne tend pas à " une normalisation des pratiquants en restreignant leur hétérogénéité potentielle au point de réduire l'inclusion à un pur discours" (Valet, op. cit., p. 165). Pourtant, dans le cadre de la Fédération française handisport, il existe un sport collectif pouvant être partagé: le basket fauteuil. On est là en présence d'une modalité d'intégration dite inversée, c'est-à-dire que des joueurs valides peuvent être accueillis dans des équipes de personnes en situation de handicap. La prise en compte des singularités s'opère par une classification des participants. Des points leur sont attribués selon leurs possibilités fonctionnelles, de 1 pour les personnes avec les capacités les plus réduites à 4,5 pour celles qui ont le moins de limitations fonctionnelles. Chaque équipe ne peut avoir un total de plus de 14 points à tout moment sur le terrain (au niveau international). Certains pays, dont 
la France, admettent dans leur championnat des joueurs valides classés dans une catégorie à 5 points.

Si cette classification conduit à une égalité des chances entre équipes, elle ne réduit pas les inégalités individuelles entre des joueurs aux capacités très diverses, même si cette diversité est limitée par l'exigence de respecter des règles communes et de maîtriser des techniques de déplacement, dribble, passe et tir dans un panier à plus de trois mètres. Bien des personnes avec une déficience motrice ne peuvent pas répondre à ces normes.

La méconnaissance des personnes en situation de handicap est un autre obstacle à la conception et à l'animation d'une pratique partagée. Son franchissement exige de surmonter les préjugés dont elles sont trop souvent l'objet. Et connaître les ressources pouvant être mobilisées par un joueur en fonction de sa déficience, ainsi que la façon plus ou moins personnelle dont elles peuvent être sollicitées est aussi précieux. Ce sont, par exemple pour une personne aveugle, des modalités sensorielles telles que le sens tactile, kinesthésique et auditif.

La référence à l'égalité peut être également, et paradoxalement, un obstacle à une pratique partagée. Ainsi, la différenciation de l'enseignement est parfois mal acceptée par les professeurs, au nom d'une égalité confondue avec l'égalitarisme. Or, une personne avec une déficience risque de ne pas avoir les mêmes chances de réussite que les autres si l'on s'en tient à une égalité formelle, sans des mesures compensatoires relevant d'un souci d'équité. Pour être profitable à tous et toutes, la différenciation du règlement des jeux sportifs collectifs apparaît donc indispensable. $\mathrm{Si}$, " aujourd'hui, le temps de la mixité sportive est déjà commencé [...] reste à innover dans l'adaptation de l'environnement et des règlements sportifs pour "bien accueillir" les personnes qui présentent des déficiences diverses " (Marcellini, 2005a, p. 9).

\section{Un intérêt croissant pour les jeux sportifs collectifs partagés, mais faible sur le processus des innovations}

Afin de situer notre étude, nous avons consulté et analysé les publications des éditions EP\&S, qui sont la référence professionnelle dominante dans le champ de l'éducation physique et du sport, en France. Le but était de voir le nombre d'articles de leurs revues sur le thème des jeux sportifs collectifs partagés, son évolution dans le temps et le contenu de ces articles. En outre, nous avons recherché dans les sept ouvrages ${ }^{2}$, de nature professionnelle ou scientifique, publiés par cet éditeur et portant sur le handicap, la place des pratiques partagées, et plus particulièrement dans le domaine des jeux sportifs collectifs.

Les éditions EP\&S publient la revue EP\&S. Elles ont aussi publié, sur l'enseignement de l'EPS à des enfants de trois à douze ans, la revue EPS1 jusqu'en 2013 (depuis,

2. Plus précisément:

- Pasqualini, M., et Robert, B. (dir.). (1996). Handicapés physiques et inaptes partiels en EPS.

- Ramanantsoa, M.-M., et Legros P. (dir.). (1999). Les activités physiques adaptées.

- Bilard, J., Ninot, G., et Varray, A. (dir.). (2001). Enseigner et animer les activités physiques adaptées.

- Bernard, P., et Ninot, G. (dir.). (2002). Les déficiences motrices.

- Ninot, G., et Partyka, M. (2007). 50 bonnes pratiques pour enseigner les APA.

- Bourgoin, T., Chigot X., Guyard Bouteiller F., et Lentz, S. (2014). Handicap et activité physique.

- Bernard, E., et Chigot, E. (20015). Jeux et sports collectifs. 


\section{DOSSIER}

les articles relatifs à cette tranche d'âge sont inclus dans la revue EP\&S). Nous avons examiné tous les articles de la revue EP\&S de 1990 à 2015, et tous ceux de la revue EPS1 jusqu'à sa disparition.

Deux périodes d'une durée égale sont distinguées: de 1990 à 2002 et de 2003 à $2015^{3}$. On constate que, d'une période à l'autre, on passe de l'absence totale d'article sur les jeux sportifs collectifs partagés à une présence à hauteur de $7 \%$ des articles sur le handicap (plus précisément concernant des personnes avec une déficience physique ou intellectuelle, ou encore des troubles psychiques), et même de $12 \%$ en incluant des articles méthodologiques centrés sur la gestion de l'hétérogénéité des publics, notamment lors de jeux sportifs collectifs partagés. Ces jeux sont donc I'objet d'une attention croissante, plus particulièrement pour les 3-12 ans, puisque un quart des articles sur le handicap portent alors sur les jeux sportifs collectifs partagés. On peut penser que les jeux traditionnels proposés à cette population jeune se prêtent mieux à une pratique partagée que les sports collectifs des adolescents, car ils peuvent être davantage adaptés en raison de leur codification plus souple. Concernant les ouvrages de la revue EP\&S traitant du handicap, si le premier (1996) consacre une partie à l'intégration, dont un article général sur la gestion de I'hétérogénéité en vue de pratiques partagées, dans les quatre suivants il n'est pas question de partage, hormis dans un article écrit par un professeur d'EPS (J.-P. Claude). En revanche, dans les deux livres les plus récents de la période retenue (2014 et 2015), écrits par des professeurs d'EPS et des cadres des fédérations sportives spécialisées, l'orientation vers l'inclusion est manifeste, le dernier étant centré sur les jeux sportifs collectifs partagés. Au final, il ressort de l'examen des sept ouvrages que les pratiques partagées ne sont mises en avant de façon conséquente que récemment.

Que ce soit dans les ouvrages ou les articles, lorsque les pratiques partagées sont abordées leur traitement s'attache alors au produit, c'est-à-dire aux modalités d'adaptation de l'activité, et non au processus de leur conception, important pour comprendre ce qui a permis l'innovation.

\section{Question pour guider la réflexion à venir}

Notre réflexion sera guidée par une question double: quelle est la pertinence de trois jeux sportifs collectifs innovants, conçus pour permettre à des personnes en situation de handicap et à des personnes ordinaires de les pratiquer ensemble, et quels sont les éléments favorables à la genèse de ces innovations, notamment ceux qui leur sont communs?

3. L'année 2003, déclarée Année européenne des personnes handicapées, est une année charnière dans le quart de siècle considéré. Elle se distingue, en France, par des événements qui témoignent d'une prise en compte nouvelle et marquante du handicap dans le domaine de l'EPS et du sport. C'est, notamment, la création, par le ministère en charge des sports, du Pôle ressources national sport et handicaps (PRNSH), ainsi que d'une "Mission nationale sport et handicaps ", chargée de tisser un réseau concourant à un objectif majeur assigné à tous les acteurs du sport: développer une politique sportive favorisant l'intégration des personnes en situation de handicap. C'est aussi la publication tout à fait inhabituelle, dans chacun des numéros de la revue EP\&S et de la revue EPS1, d'un ou plusieurs articles sur le thème de l'EPS et du handicap durant cette année-là. 
Cette réflexion s'appuyant sur des jeux différents, nous aurons préalablement à décrire leurs architectures respectives, à en saisir le caractère plus ou moins innovant, puis à présenter le processus qui y a conduit.

\section{CADRE CONCEPTUEL ET MÉTHODOLOGIQUUE}

Notre propos est ici de préciser le sens de concepts présents dans l'article et d'exposer la méthode privilégiée pour l'étude des jeux retenus.

\section{Concepts}

Emprunté à Pierre Parlebas, le concept de jeu sportif se présente comme "une situation motrice d'affrontement codifiée, dénommée "jeu" ou "sport" par les instances sociales " (op. cit., p. 112). Le jeu sportif, défini par " son système de règles qui en détermine la logique interne " (ibid.), est dit collectif lorsqu'il mobilise des interactions motrices de coopération, entre partenaires, et d'opposition à des adversaires. II comprend deux types de jeux: des jeux sportifs traditionnels, inscrits dans un patrimoine culturel souvent ancien, et des jeux sportifs institutionnels, ou sports, régis par des règlements précis sous l'égide d'une institution reconnue, en l'occurrence une fédération sportive.

Pour sa part, le concept de pratique partagée désigne une pratique commune à des personnes en situation de handicap et à des personnes ordinaires, dans un même espace et un même temps, jusqu'à leur regroupement dans une même équipe. En France, le mot partagé tend à être préféré à mixte, qui serait également pertinent. Ainsi, les deux fédérations sportives du sport scolaire (I'Usep et l'UNSS) I'ont adopté, de même que deux importantes fédérations multisports (I'Ufolep et la FSGT).

Quant au concept d'innovation, il ne se confond pas avec celui d'invention. La distinction est apportée dès 1801 par Quatremère de Quincy, à propos des beauxarts et de l'architecture, dans son Encyclopédie méthodique (citée par Guillerme, 2008), puis précisée avec Joseph Schumpeter (1999) et les économistes industriels. Nous retiendrons que l'innovation suppose une invention, mais aussi qu'elle soit mise en œuvre, se diffuse en étant adoptée dans un milieu social (A/ter, 2000), et qu'elle apporte une amélioration à l'existant, ainsi que le souligne Huberman dans le domaine de l'éducation (1973).

II sera ici question d'innovation sociale, entendue par le Crises (Centre de recherche sur les innovations sociales) comme " une intervention initiée par des acteurs sociaux, pour répondre à une aspiration, subvenir à un besoin, apporter une solution ou profiter d'une opportunité d'action afin de modifier des relations sociales, de transformer un cadre d'action ou de proposer de nouvelles orientations culturelles " (2009, p. 4). Elle se distingue d'autres types d'innovations: "L'innovation sociale est inclusive et créatrice de mieux être, elle est souvent le fruit d'un travail collectif où le moteur n'est pas uniquement, contrairement à l'innovation destructrice, la brevetabilité, donc la plus-value, mais la partageabilité et la libre transférabilité. " (Von Bülow, 2012, p. 10). 


\section{DOSSIER}

\section{Méthode}

L'étude de cas est une méthode qualitative privilégiée pour notre questionnement. Appelant une connaissance approfondie de l'objet étudié, elle est adaptée à sa description détaillée et à la compréhension des processus en jeu dans sa construction (Gagnon, 2012). Selon Yin (1994), elle convient bien pour des recherches où I'on s'interroge sur le comment et le pourquoi d'évènements sur lesquels le chercheur n'a pas de contrôle. Elle est aussi particulièrement appropriée pour identifier des similarités entre plusieurs cas. De ce point de vue, Howard S. Becker (2016) valorise le raisonnement analogique pour la recherche en sciences sociales, la comparaison de cas singuliers permettant une réflexion plus générale.

Les jeux sportifs collectifs ont été choisis selon trois critères principaux: ils nous paraissaient présenter les caractéristiques d'une innovation, ils se distinguaient plus ou moins les uns des autres par leur degré de codification et d'institutionnalisation, ce qui pouvait enrichir une étude de cas comparative, et ils étaient suffisamment bien documentés pour que l'on puisse en connaître la genèse.

Le baskin est abordé en grande partie à partir de la thèse soutenue par Alexy Valet (2013), tandis que l'étude des deux autres jeux repose sur des éléments nombreux et pour beaucoup méconnus auxquels l'accès a été facilité du fait que l'auteur de cet article fut impliqué dans I'histoire de ces jeux. II n'en est pas l'inventeur, mais, de par sa position institutionnelle à l'époque (formateur au Cnefei - Centre national d'études et de formation pour l'enfance inadaptée -, devenu l'INSHEA), il a pu repérer leur émergence, l'accompagner et contribuer à leur diffusion.

Par ailleurs, nous nous appuierons succinctement sur des entretiens conduits auprès de jeunes aveugles ayant vécu l'un ou l'autre des jeux adaptés à une déficience visuelle, et auprès d'un professeur d'EPS impliqué dans I'un d'eux.

\section{ARCHITECTURE DE TROIS JEUX SPORTIFS COLLECTIFS ROMPANT AVEC L'INDIFFÉRENCIATION DU RËGLEMENT}

II s'agit là de décrire les aspects matériels et réglementaires des trois jeux retenus.

\section{La thèque adaptée aux enfants aveugles}

À la différence du base-ball, sport dont elle est proche, la thèque, jeu traditionnel, n'est pas institutionnalisée. Non définies par une fédération sportive, ses règles sont donc susceptibles d'être modifiées, mais un élément essentiel réside dans l'obligation de parcourir le plus rapidement possible un circuit constitué de bases matérialisées sans se faire éliminer par les adversaires.

Les adaptations ont été conçues à l'origine pour permettre à Yann, un enfant aveugle âgé de 10 ans, de participer au jeu avec des camarades voyants dans une salle. 
Schéma : Thèque adaptée aux enfants aveugles

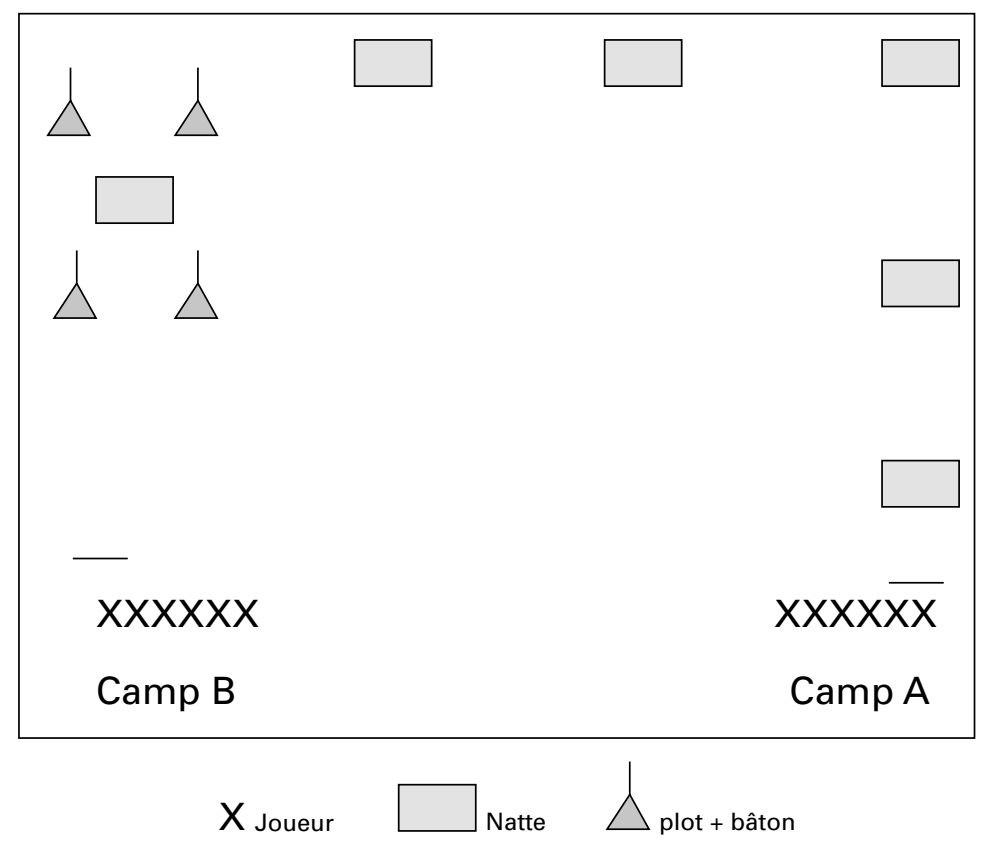

Dans l'équipe A, équivalente à celle des lanceurs au base-ball, le parcours à effectuer est en forme de $\mathrm{L}$. Ses bases sont matérialisées par des nattes, réparties à intervalle régulier dans la salle. Les unes sont disposées le long d'un mur, jusqu'à une natte placée dans un angle; les autres sont à la suite, le long du mur perpendiculaire au précédent. Yann peut ainsi les localiser en s'informant tactilement avec ses pieds, tandis que leur situation le long de murs lui permet de se repérer en les suivant avec ses mains. Un signal sonore est émis par un co-équipier qui agite des grelots quand il estime que Yann doit s'arrêter sur une base pour ne pas être éliminé.

Dans l'équipe $B$, les uns après les autres, les joueurs vont rapidement vers l'endroit où sont disposés 4 bâtons insérés dans des cônes pour y enfiler 4 anneaux puis revenir au point de départ. Yann effectue une tâche différente : il doit aller ramasser 4 anneaux posés sur une natte (éventuellement à l'intérieur d'un petit cerceau pour faciliter leur localisation) et les ramener à son point de départ. Pour se diriger vers la natte, il est guidé par des indications sonores, par exemple des frappés de mains d'un partenaire situé au niveau de la natte à atteindre.

Pour que les chances de victoire des deux équipes soient équilibrées, on peut aussi accorder à l'enfant aveugle le double de points pour un même résultat. Ou bien un élève voyant de l'autre équipe a les yeux bandés. 


\section{DOSSIER}

\section{Le basketball adapté aux personnes déficientes visuelles}

Certaines règles sont communes à tous les joueurs, d'autres non.

\section{Des règles communes}

- Tout joueur en possession du ballon doit le signaler immédiatement en disant " j'ai ».

- Les passes doivent être exécutées avec rebond.

\section{Des règles différenciées}

- Quand le jeu est pratiqué par des personnes malvoyantes et des personnes aveugles, un joueur malvoyant ne peut s'emparer du ballon que lorsque celui-ci a rebondi au moins deux fois lors d'une passe.

- Chaque joueur dribble en fonction de ses capacités. Certains dribblent à une main, d'autres à deux mains.

- Un joueur malvoyant n'a pas le droit de contrer le tir d'un adversaire aveugle.

- Le décompte des points est variable selon les possibilités visuelles des joueurs.

\section{Le baskin}

Le terme baskin correspond à l'abréviation de basket intégrant, ou basket inclusif, indiquant la co-participation de personnes ordinaires et d'autres ayant divers types de déficience, physique ou intellectuelle, au sein d'une même équipe. La seule condition requise pour pouvoir prendre part à cette activité est d'être capable d'effectuer un lancer de balle dans un panier selon une trajectoire parabolique, que le panier soit haut ou bas, que la balle soit grosse ou petite, que le joueur soit proche ou éloigné. Les autres habiletés motrices (course, dribble, passe) ne sont donc sollicitées que pour les joueurs qui s'en révèlent capables.

\section{Adaptation du matériel}

Six paniers de basket sont présents: deux paniers normaux, plus deux paniers latéraux plus bas (I'un à $2 \mathrm{~m}$, l'autre à $1 \mathrm{~m}$ ), de chaque côté de la ligne médiane. Le nombre total de paniers où l'on peut marquer des points est donc de 6 (3 par équipe). - II est possible d'utiliser un ballon de taille et de poids différents lors d'un tir dans un panier latéral.

\section{Adaptation de l'espace}

Chaque équipe peut choisir à tout moment parmi deux zones d'attaque: une zone standard et une zone latérale marquée sur le sol.

\section{Adaptation des règles}

Les rôles des joueurs sont différenciés en fonction de leurs capacités motrices fonctionnelles. Cinq rôles sont ainsi distingués, identifiables par un numéro inscrit sur le maillot, allant de 1 à 5 , définissant pour chacun d'eux des droits particuliers: interdiction de défendre sur un joueur de rôle inférieur; marge de tolérance variable concernant les fautes de dribble selon le rôle occupé; nombre de tirs limité par temps de jeu pour certains joueurs, etc. En outre, une fonction de tutorat permet d'aider certains coéquipiers si besoin. 


\section{Des jeux plus ou moins innovants}

Employer le terme d'innovation à propos des trois jeux présentés peut être discuté à partir d'une distinction opérée, à la suite de Christensen (1997), entre des innovations radicales, dites de rupture car créant un objet à l'identité et à l'usage tout à fait nouveaux, comme le téléphone versus le télégraphe, et les innovations incrémentales, ou de soutien, qui renforcent ou améliorent l'existant sans constituer un bouleversement, telle la génération $4 \mathrm{G}$ de réseaux mobiles à la place de la $3 \mathrm{G}$. Parce qu'ils comportent des règles différenciées selon les capacités fonctionnelles des joueurs, les trois jeux sportifs collectifs étudiés introduisent une rupture fondamentale avec la logique sportive, mais leurs nouveautés sont de degrés différents, ainsi que le montre l'examen des deux sports collectifs transposés du basketball. Alors que la profonde transformation des conditions de jeu du baskin fait de ce sport un objet à l'identité originale, tel la thèque adaptée, le basketball adapté garde globalement les traits fondamentaux du sport de référence. Seul le distingue un règlement différencié, mais qui ne l'est pas lors des compétitions contre des adversaires voyants, organisées dans le cadre de I'UNSS (Union nationale du sport scolaire). De ce point de vue, cette adaptation du basketball relève donc davantage d'une innovation incrémentale que d'une innovation radicale.

\section{LES TROIS JEUX DU POINT DE VUE DE LEUR DYNAMIOQUE}

Nous envisageons ici le processus qui a conduit à l'invention et à la diffusion des trois jeux.

\section{Leur élaboration}

\section{La thèque adaptée}

La première trace écrite de cette thèque adaptée apparaît en 2001 dans le mémoire présenté par Dominique Germez, professeur des écoles, pour l'examen lui permettant de devenir enseignante spécialisée, mais son invention s'inscrit dans la continuité d'une adaptation de ce jeu à des enfants avec une déficience motrice.

En formation au Cnefei pendant une année, Germez a pu, lors d'un stage en établissement scolaire, expérimenter des adaptations avec une classe comportant un enfant aveugle. À cet effet, elle s'est appuyée sur un jeu de thèque adapté à des enfants avec une déficience motrice, inventé à la fin des années quatre-vingt par Jean Chevrier, alors professeur d'EPS à l'école de l'hôpital de Saint-Maurice. Son invention avait été repérée par les formateurs en EPS du Cnefei. Ils entreprirent de la faire connaître, notamment à travers l'article d'un livre dirigé par un formateur du Cnefei (Chevrier, 1996) et un film réalisé par le département audiovisuel de cet établissement, en 1992. Germez eut l'occasion de le visionner, de lire l'article sur ce jeu et d'être accompagnée par un formateur pour l'adapter à un enfant aveugle.

\section{Le basketball adapté}

Les adaptations ont été conçues par Fred Gomez, professeur d'EPS. C'est à I'Inja (Institut national des jeunes aveugles - Paris), où il enseignait, qu'il les a mises au 


\section{DOSSIER}

point dans les années quatre-vingt. II a fondé son invention sur une observation attentive de l'activité de ses élèves et sur un pari éducatif ambitieux accompagné de solides connaissances sur la cécité, dues à la fonction d'instructeur en locomotion qu'il assurait à I'Inja. Cette fonction le conduisait à enseigner à de jeunes aveugles comment se déplacer dans l'environnement, ce qui suppose des connaissances précises concernant les modalités sensorielles mobilisables par des personnes aveugles et la façon de les mobiliser. On a évoqué le toucher et l'audition en se limitant, pour cette dernière, aux sources sonores directes, mais il existe aussi des sources sonores indirectes, qui permettent la détection des masses et des obstacles sans utilisation du toucher, par écholocation, c'est-à-dire l'utilisation de l'écho produit par un son. Cette modalité sensorielle était sollicitée par Gomez dans son enseignement du basketball.

\section{Le baskin}

Le baskin n'aurait sans doute pas vu le jour, à Cremone, dans le nord de I'Italie, sans la naissance de Marianna Bodini, en 1988, avec une infirmité motrice cérébrale. Sa déficience conduit son père, Antonio Bodini, à rencontrer Adriano Ferrari, médecin rééducateur, héritier de Adriano Milani Comparetti, pour qui « il ne peut y avoir de réhabilitation motrice sans réhabilitation sociale " (Valet, op. cit., p. 296). De ce contact, Bodini retient une conception de la santé qui met l'accent sur la qualité de vie globale du sujet et sur l'intégration.

Influencé par sa connaissance d'une activité basketball ouverte, dans la commune de Soresina, à des jeunes dont certains présentent des déficiences, Bodoni propose à Fausto Capelini, professeur d'EPS du collège où Marianna est scolarisée, de mettre en place dans ce collège un jeu de basketball partagé mais qui soit un "vrai sport", dans lequel les joueurs en situation de handicap ne seraient pas assistés, selon lui, comme à Soresina. Ils engagent alors un travail collectif impliquant plusieurs personnes. Beaucoup d'échanges informels vont faire germer progressivement, entre 2001 et 2005, les idées de rôles différenciés, de cibles diverses et de zones protégées, pour aboutir à une architecture du baskin formalisée. Cette architecture va continuer d'évoluer, l'identité du baskin n'étant en fait qu' « une forme provisoirement stabilisée par un consensus intersubjectif " (Valet, op. cit., p. 296) entre des acteurs qui ont développé leur invention par tâtonnements et adaptations en s'appuyant sur l'observation des habiletés fonctionnelles des joueurs sur le terrain.

Le travail d'élaboration du baskin a été animé avec la conviction que la réussite de l'intégration passe par une structuration règlementaire du jeu de façon à permettre à chaque joueur d'exprimer au mieux ses potentialités lors d'un vrai défi sportif, ce qui invite à déjouer le piège de l'injustice, dont serait victime un joueur handicapé face à un adversaire valide si le règlement n'était pas aménagé, et celui de l'assistanat, où les personnes valides seraient surtout cantonnées dans un rôle d'aide à leurs partenaires handicapés.

\section{Leur diffusion}

Le jeu de thèque adapté aux enfants aveugles s'est fait connaître par un article de la revue EPS (Garel, 2003), et on le trouve aujourd'hui sur le site web de I'Inspection 
académique du Calvados (2008). Toutefois, on manque d'informations sur l'extension de sa pratique effective. Vu le petit nombre d'élèves aveugles à l'école ordinaire, il est raisonnable de penser que sa pratique est restreinte.

Pour sa part, le basketball adapté a été repéré, comme la thèque de Chevrier, par les formateurs en EPS du Cnefei. Ils l'ont présenté pour la première fois en 1986, lors des journées d'études regroupant les enseignants d'EPS français intervenant auprès d'élèves déficients visuels. Les actes de ces journées (Gomez, 1987) ont permis d'élargir la connaissance de cette expérience au-delà d'un petit cercle de spécialistes. Par la suite, ce basketball adapté a donné lieu à des communications dans des colloques, des publications d'articles (Garel et Siros, 1992; Gomez et Siros, 1996; Garel, 2002), et un livre présentant une modélisation de la démarche d'enseignement (Garel, Siros et Gomez, 1992). Aujourd'hui, on en trouve les principaux éléments dans un document sur l'enseignement de I'EPS aux élèves déficients visuels, élaboré par le groupe EPS adaptée de l'académie de Versailles et repris sur les sites web des académies d'Orléans-Tours et de Grenoble.

Concernant la pratique effective du basketball par des personnes déficientes visuelles, peu d'informations sont disponibles. On peut citer le témoignage d'un professeur d'EPS (Frank, 2006) et l'engagement régulier d'une équipe de I'Inja dans le sport scolaire ordinaire. Elle est composée uniquement de joueurs malvoyants, excluant ceux qui sont aveugles, et la norme des personnes voyantes prévaut, puisque ce sont les règles standard du basketball qui sont alors adoptées. C'est là un sport dont on ne connaît pas de prolongements hors de l'école.

Quant au baskin, sa reconnaissance passe notamment par des présentations en colloque, une thèse (Valet, op. cit., 2013), un article (Valet, 2015), et des modules

de formation à l'université. II se distingue des deux activités précédentes par une institutionnalisation plus marquée. En 2005 a eu lieu, en Italie, un premier championnat scolaire au niveau départemental. Dès 2010-2011, I'association baskin, créée en 2006, a structuré de façon plus formalisée son extension territoriale jusqu'au niveau national. En France, le baskin est pratiqué à l'association Orea, à Nantes, dans le cadre d'une fédération multisports (I'Ufolep). Fin 2012, on comptait environ 3000 pratiquants, dont le quart de joueurs en situation de handicap.

\section{REGARD SUR LA PERTINENCE DES JEUX}

S'interroger sur la pertinence des jeux étudiés implique en premier lieu de se demander dans quelle mesure ils permettent effectivement des pratiques partagées. II s'agit aussi de voir si les modalités de différenciation des règles ne sont pas sans risque et n'appellent pas de précaution particulière, si les adaptations proposées sont satisfaisantes pour les joueurs, avec une déficience ou non, et si ces jeux sont tellement pertinents, du point de vue de l'inclusion, qu'ils devraient supplanter les pratiques entre soi.

\section{L'accès à des pratiques partagées}

Les jeux sportifs collectifs mobilisent des ressources qui sont plus ou moins limitées chez les personnes avec une déficience. Celle de nature cognitive, sollicitées pour 


\section{DOSSIER}

les prises d'information et de décision dans un environnement incertain, pénalise les participants ayant une déficience intellectuelle. L'incertitude liée à l'environnement, aux déplacements des joueurs et du ballon met en difficulté les pratiquants déficients visuels. Et les joueurs avec une déficience motrice sont plus ou moins entravés dans la réalisation de leurs actions. Le fait que, malgré ces manques, il soit possible d'accéder à des pratiques partagées et d'y persévérer est un élément qui, de ce point de vue, montre la pertinence des jeux considérés.

Toutefois, le partage n'est pas identique dans ces trois jeux. Alors que tous les joueurs, avec une déficience ou non, sont dans une même équipe pour pratiquer la thèque adaptée et le baskin, il n'en va pas ainsi dans le basketball adapté. Dans ce cas, nous avons, en cours d'EPS à I'Inja, des équipes comprenant à la fois des élèves malvoyants et des élèves aveugles, ou bien, lors des championnats scolaires, une équipe composée uniquement de joueurs malvoyants opposés à des adversaires voyants. Dans ce dernier contexte, on a bien le partage d'une même activité, mais dans une situation d'opposition, et non de coopération/opposition comme dans les deux autres jeux.

Il faut comprendre que si les personnes malvoyantes peuvent arriver à jouer au basketball avec des personnes voyantes, moyennant des adaptations, c'est en revanche plus problématique pour celles qui sont aveugles. On touche là aux limites du partage. En témoigne la présentation et l'analyse que fait un professeur d'EPS de son expérience avec un élève aveugle accueilli en classe ordinaire. Cet élève a bien participé au cycle de basketball, mais pas aux rencontres entre équipes. II n'a pu apprendre que des gestes techniques (Frank, 2006).

\section{La différenciation des règles}

On constate que les règles ne sont pas fixées de la même façon pour les deux sports dérivés du basketball. Pour le baskin, un rôle est affecté à chaque joueur, qui définit ses droits pour l'ensemble des éléments du jeu: dribble, tir, défense ou non sur l'adversaire, etc. Mais il n'y a que cinq rôles. Pour sa part, le basketball adapté est régi par une modalité de différenciation plus souple, puisque à chaque joueur peuvent être attribués des droits particuliers. D'un côté, on a un risque, relatif, de rigidité, de l'autre on s'expose à une individualisation extrême, qui, faute de critères clairs, peut amener les joueurs à mettre en cause le bien-fondé de ce qui est accordé à l'un et pas à l'autre. Pour ne pas être soupçonné de décisions subjectives et injustes, l'enseignant/éducateur/entraîneur doit donc fonder explicitement les inégalités de droit au nom de l'équité. Pour Rémi Roussel, professeur d'EPS qui a succédé à Fed Gomez à I'Inja, il est par conséquent nécessaire d'impliquer les joueurs pour aboutir à un consensus.

\section{Des satisfactions partagées?}

Des acteurs de ces jeux innovants font entrevoir ce qui est éprouvé par les pratiquants. Un entretien avec un groupe d'élèves aveugles scolarisés en classe de première à I'Inja montre leur fierté de réaliser en basketball des performances incroyables pour beaucoup. L'un d'entre eux: "J'essaie d'expliquer le basket (pratiqué par des aveugles - NDLR) un petit peu, alors on s'est foutu de ma gueule; "ce n'est pas 
possible, des aveugles au basket, ce n'est pas possible, impensable." Ce qui est grave, c'est que c'est même dans un milieu d'aveugles; donc cela prouve qu'eux ne savent pas. "De son côté, Rémi Roussel, leur professeur d'EPS, souligne la satisfaction de joueurs malvoyants engagés dans le championnat scolaire où ils étaient opposés à des adversaires voyants: "I/s ont gagné de temps en temps. Quand la victoire arrive, ça a un sacré goût!"

Quant aux valides, la pertinence de ces jeux tient notamment à ce qu'ils puissent y trouver des défis à leur mesure, sans être réduits au sentiment de faire une bonne action (Valet, op. cit.) en jouant avec des handicapés et en les faisant jouer, parfois sous l'injonction de l'animateur: "passez-lui [leur] la balle! »

L'exemple de la thèque avec Yann, l'enfant aveugle, témoigne d'une satisfaction partagée à partir du moment où l'enseignant ne s'est plus focalisé sur lui. Yann: "Pour moi, tout était très bien .» Quant à l'avis général de ses camarades voyants, il est exprimé par l'un d'entre eux: "Au début, ce n'était pas très bien car tout était fait pour Yann. C'était trop facile pour nous. [Ensuite] le jeu de la thèque c'était très bien car nous avons tous pu jouer de la même façon. " (Germez, op. cit. p. 27).

Pour les joueurs avec une déficience comme pour les autres, un niveau d'exigence adapté conditionne leur engagement dans l'activité et leur satisfaction. Dans ce sens, la pertinence des pratiques partagées nécessite de se frayer une voie entre l'écueil d'une normalisation qui exclut les personnes les moins performantes et l'écueil d'une bienveillance excessive, telle qu'elles sont confrontées à des situations insuffisamment exigeantes, qui, ne leur posant pas de défi à la hauteur de leurs possibilités et du plaisir attendu, les assignent plus ou moins à leur différence.

\section{Pratiques partagées versus pratiques entre soi}

Doit-on déduire de la réussite de pratiques partagées que les activités rassemblant uniquement des personnes ayant des déficiences sont à écarter? En fait, l'intérêt de jeunes déficients visuels pour le basketball peut parfaitement se conjuguer avec celui pour le torball, sport collectif qui leur est propre, pourvoyeur d'autres satisfactions, notamment parce qu'il est plus accessible, d'un apprentissage moins exigeant, plus répandu, ouvert à des rencontres dans un cadre fédéral. Ces deux sports constituent chacun une référence culturelle distincte et attractive pour des raisons différentes. La référence représentée par le torball est largement partagée. Elle marque l'appartenance à la communauté des personnes avec une déficience visuelle, mais elle signe un handicap. Pour sa part, le basketball est une pratique trop méconnue parmi cette population pour être une référence identitaire commune. D'un autre côté, elle inscrit plus ou moins dans la norme les personnes handicapées qui s'y livrent. En montrant leur capacité à y accéder, elles atténuent leur différence. Un effort de lucidité est nécessaire pour ne pas verser dans l'apologie idéologique d'une mixité sans limite. Anne Marcellini a montré l'intérêt, du point de vue même de ses acteurs, d'une pratique entre sportifs en fauteuil roulant (Marcellini, 2005b). De son côté, Didier Seguillon a souligné que le sentiment d'appartenance des personnes sourdes à une culture originale les conduisait à souhaiter être ensemble pour des activités communes (Seguillon, 2015). Pour sa part, Michèle Sarloute, ancienne championne de torball, exprime ce qu'elle apprécie dans des activités entre soi: 


\section{DOSSIER}

"Se retrouver entre personnes handicapées, entre femmes, a des avantages. On a en effet le désir d'être parfois entre soi, car on a des intérêts communs et on est souvent confrontées aux mêmes problèmes [...]. Évoluer ou non avec des valides relève d'un choix personnel jamais arrêté, fonction des opportunités. " (Sarloute, 2015, p. 44).

\section{FACTEURS DE RÉUSSITE DES INNOVATIONS}

En examinant l'histoire des trois jeux sous l'angle des conditions qui en ont permis I'élaboration et la diffusion, nous entendons dépasser les cas particuliers pour dégager, autant que possible, des conditions qui leur sont communes et que l'on rencontre dans la littérature sur l'innovation.

À l'origine des trois jeux, on trouve l'engagement d'acteurs (Taylor, 1970) qui ont eu un rôle décisif: pour la thèque, Chevrier puis Germez; pour le basketball adapté, Gomez, et Bodini avec Capellini pour le baskin. L'engagement est mû par des idées hors cadre (Lucidarme, 2013), qui rompent avec des schémas de pensée habituels. Concernant les jeux sportifs collectifs, il a fallu se mettre à distance des jeux sportifs connus, ne pas les considérer comme des objets culturels immuables. II a fallu aussi briser le principe de l'homogénéité des équipes sportives et de l'indifférenciation des règles, questionner la conception de l'égalité qui préside à une organisation des compétitions, s'émanciper des représentations communes qui sont liées aux personnes en situation de handicap et imaginer, par exemple, que des personnes aveugles puissent jouer au basketball.

S'aventurer sur des chemins inconnus implique une prise d'initiative et de risques (Cloutier, op. cit. ; Lapeyronnie, 2014), l'engagement dans une voie à l'issue incertaine bousculant le confort des habitudes. Et l'initiative est impossible sans une autonomie suffisante (Cloutier, op. cit. ; Lapeyronnie, op. cit.). Chevrier et Gomez enseignaient dans des établissements spécialisés auprès de populations très particulières, aux déficiences souvent importantes. Ils étaient amenés à inventer, avec une marge de liberté d'autant plus grande que les contenus et les démarches d'enseignement avec ces élèves singuliers étaient en grande partie à construire.

Innover suppose également l'ouverture à des partenaires (Lapeyronnie, op. cit.), selon une démarche qui peut valoriser la cooptation (Taylor, op. cit.), en particulier dans le cas de l'innovation sociale. Ainsi, aux premiers acteurs du projet autour du baskin sont peu à peu venus s'agréger d'autres personnes. Au-delà, des partenariats institutionnels ont été noués. Au Cnefei, entre 1986 et 1993, six regroupements nationaux des enseignants spécialisés de I'hexagone, dont deux uniquement sur les jeux sportifs collectifs, ont contribué à les faire connaître. À noter que les formateurs du Cnefei, organisateurs de ces regroupements, n'avaient pas de statut leur conférant une quelconque autorité administrative sur les participants. Les relations égalitaires (Taylor, op. cit.) ont sans doute concouru à une dynamique conviviale, source d'échanges maintenus après ces temps en commun, à l'origine d'un livre collectif où les jeux sportifs collectifs occupent une bonne place (Garel, 1996), et dont Germez s'est inspirée pour concevoir la thèque adaptée. La convivialité, et plus généralement le climat humain, sont 
mentionnés par A. Valet comme des éléments qui ont favorisé l'élaboration du baskin (Valet, op. cit.,).

Par ailleurs, la dimension cognitive peut être un élément important de l'innovation. Des savoirs sont requis (Cloutier, op. cit.), dont certains sont plutôt théoriques, extérieurs au champ de la pratique professionnelle. Ici, ils relèvent par exemple du médical, pour les précautions à prendre face à une pathologie donnée, et de la physiologie. Ainsi, des adaptations ont été nourries par la connaissance des modalités de prise d'informations palliant partiellement une perte de vision.

Des savoirs professionnels, explicatifs et opératifs, en grande partie inscrits dans le champ de la didactique, sont également présents. Ils sont d'ordre général, ou plus spécifique aux activités physiques et sportives, comme ceux élaborés par Famose (1990), par exemple sur les "exigences " d'une tâche, encore dites "difficultés objectives ", car propres à une tâche indépendamment des capacités du sujet qui l'effectue. Ces savoirs sont croisés avec la connaissance des capacités du sujet qui effectue la tâche, selon une démarche d' « ingénierie pédagogique » visant à adapter l'enseignement à chacun et chacune.

Les savoirs d'action sont d'une autre nature. Ce sont des savoir-faire explicités, résultant d'une décontextualisation/conceptualisation de savoir-faire implicites, liés à une action et son contexte (Pastré, 1999). La mise en mots de la démarche d'enseignement du basketball à des personnes déficientes visuelles à travers un livre (Garel, Siros et Gomez, op. cit.) participe de ce type de savoir, utile pour la diffusion de cette expérience et son appropriation par d'autres acteurs.

La dimension cognitive se manifeste également par la réflexivité (Lapeyronnie, op.cit.), c'est-à-dire par l'analyse rétrospective de l'activité par ses acteurs. Elle est particulièrement à l'œuvre dans l'élaboration du baskin, certes bien formalisé en 2005, mais toujours objet de réflexions pour le faire évoluer.

La réflexion des acteurs accorde la priorité aux situations (Lapeyronnie, op. cit.). L'innovation confrontant celles et ceux qui s'y engagent à affronter l'inconnu, à se risquer dans des situations complexes et changeantes, il serait vain de vouloir leur appliquer strictement des solutions empruntées à d'autres contextes, des théories trop déconnectées de la pratique, ou des outils didactiques et pédagogiques donnant lieu à des prescriptions relevant d'un prêt-à-porter inajustable à une réalité singulière. On suivra plutôt le courant de la didactique professionnelle (Pastré, Mayen et Vergnaud, 2006) pour avancer que la compétence de l'innovateur, dans le domaine de l'intervention en EPS et en sport, se fonde pour beaucoup sur l'analyse des situations de terrain, en l'occurrence de jeu, et de l'activité déployée par les sujets engagés dans ces situations.

Comme, généralement, on n'invente pas à partir de rien, la transmission des expériences et des savoirs (Delay, 2006) est importante. L'invention de la thèque adaptée a été permise par la connaissance que Germez a pu avoir de l'expérience de Chevrier et des savoirs qu'elle révèle. Publications, films et formations ont concouru à cette transmission.

Enfin, les individus et les collectifs engagés dans une innovation doivent disposer des ressources suffisantes pour penser, mettre en œuvre et diffuser leur projet. Des ressources sont donc nécessaires au renforcement des acteurs (Lapeyronnie, 


\section{DOSSIER}

op. cit.). À Cremone, le démarrage du projet a nécessité la mise à disposition d'un gymnase et d'un créneau horaire. Pour le basketball adapté et la thèque adaptée, c'est moins l'invention elle-même et ses débuts de mise en œuvre qui ont appelé des ressources particulières que leur diffusion. Les six regroupements nationaux entre 1986 et 1993, financés par le ministère de l'Éducation nationale, le livre sur l'enseignement des sports collectifs aux personnes aveugles, financé par I'Inspection générale d'EPS, et les crédits alloués à la réalisation de films réalisés par le Cnefei ont participé à une connaissance élargie des activités innovantes.

\section{CONCLUSION}

L'étude des conditions ayant permis l'émergence et le développement de trois jeux sportifs collectifs nous a conduit à dépasser les particularités des cas étudiés et à identifier des facteurs de réussite qui leur sont en grande partie communs. On les retrouve dans des situations d'innovation relevant de domaines différents et I'on peut faire l'hypothèse qu'ils sont plus ou moins mobilisables dans d'autres contextes.

À côté de ces aspects communs, on a pu constater des particularités. En introduisant une différenciation des règles selon les capacités fonctionnelles des participants, les trois jeux étudiés rompent avec l'organisation sportive dominante, mais ils se distinguent les uns des autres sur plusieurs points. D'abord, par leurs niveaux de codification et d'institutionnalisation. Dans le cas de la thèque, jeu traditionnel, donc non institutionnalisé, la souplesse réglementaire apparaît telle que l'individualisation des droits peut être très forte. On a une même souplesse dans le basketball adapté aux personnes déficientes visuelles, du moins tant qu'il ne donne pas lieu à des rencontres dans le cadre d'une fédération sportive, puisque alors c'est le règlement du basketball ordinaire qui prévaut. L'organisation dominante du sport s'accommode mal d'une opposition entre des joueurs aux droits différents. C'est le défi de concilier individualisation et institutionnalisation que s'attache à relever le baskin, en procédant à une différenciation significative, quoique limitée à cinq catégories dans lesquelles doivent s'inscrire les joueurs.

On peut aussi distinguer ces jeux selon leur degré de rupture par rapport aux jeux sportifs collectifs de référence. Les conditions matérielles et réglementaires de la pratique du baskin et de la thèque adaptée en font de véritables innovations de rupture. Quant au basketball adapté, on a bien une rupture, tenant à la différenciation du règlement, mais l'identité de ce sport n'est pas fondamentalement remise en cause. II s'agit d'une innovation incrémentale plutôt que de rupture. On remarquera que les trois innovations rapportées ont été conçues dans le champ scolaire, plus ouvert au détournement des normes sportives que l'univers fédéral, fortement institutionnalisé.

Les trois jeux résultent d'une invention et se sont diffusés, mais inégalement. Peu pour la thèque et le basketball adapté, pour partie en raison de la relativement faible prévalence de cette déficience et plus particulièrement de la cécité; plus largement pour le baskin. À des degrés divers, ils ont donc ces caractéristiques fondamentales de l'innovation que sont l'invention et la diffusion; et aussi l'apport d'une amélioration 
à l'existant, puisqu'ils constituent un progrès, en termes d'opportunité d'accès à une pratique sociale partagée.

S'appliquer à faire partager des jeux sportifs collectifs à des personnes en situation de handicap et à des personnes ordinaires ne va pas de soi. C'est un défi d'autant plus ambitieux et difficile à relever que l'on s'attache à un partage suffisamment riche de bénéfices pour tous les joueurs. D'ailleurs, ce point mériterait une recherche pour établir plus solidement la réalité et les conditions des bénéfices retirés par tous les participants à une activité commune.

Maintenant, que les innovations soient à encourager dans le sens des pratiques partagées ne disqualifie pas une pratique entre soi. Elle peut être privilégiée si elle est davantage à la mesure de la personne, plus sécurisante et protectrice (Compte et Marcellini, 2005), et si les pratiquants y trouvent une proximité identitaire qui les satisfait. À la place ou en plus d'une pratique partagée, elle n'est pas sans pertinence. Plutôt que de cristalliser les oppositions, ne convient-il pas d'exploiter les complémentarités, de faire en sorte que chaque personne puisse circuler d'un contexte de pratique à l'autre, selon ses besoins et ses désirs, pour y trouver matière à son accomplissement personnel et à une participation sociale optimale?

\section{Bibliographie}

Alter, N. (2000). L'innovation ordinaire. Paris: PUF.

Becker, H. S. (2016). La bonne focale. De I'utilité des cas particuliers en sciences sociales. Paris: La Découverte.

Bui-Xuân, G., et Mikulovic, J. (2006). L'intégration par le sport des personnes handicapées mentales. Les Cahiers du sport adapté, 8, 40-48.

Chevrier, J. (1996). La thèque. In J.-P. Garel (dir.), Éducation physique et handicap moteur (pp. 143-158). Paris: Nathan.

Christensen, C.M. (1997). The Innovator's Dilemma. When New Technologies Cause Great Firms to Fail. The Management of Innovation and Change. Boston: Harvard Business School Press.

Cloutier, J. (2003). Qu'est-ce que l'innovation sociale? Les Cahiers du CRISES, Collection Études théoriques, no ET0314. Montréal: Université du Québec.

Commission EPS et handicap. (2008). EPS à l'école et handicap moteur ou sensoriel. Inspection académique du Calvados. http://eps21.ac-dijon.fr/spip.php?article54

Compte, R., et Marcellini, A. (2005). Études des représentations du sport d'élite chez les entraîneurs et les athlètes des équipes de France de la FFSA. Les Cahiers du sport adapté, 2, 79-99. Paris: FFSA.

Crises (Centre de recherche sur les innovations sociales). (2009). Rapport annuel des activités scientifiques 2008-2009. Montréal : Université du Québec. <http:// crises.uqam.ca/upload/files/rapports-annuels/Rapport_annuel_08-09.pdfs

Delay, B. (2006), La transmission des savoirs dans I'entreprise. Construire des espaces de coopération entre les générations au travail. Informations sociales, 134(6), 66-77. http://www.cairn.info/revue-informations-sociales-2006-6-page-66.htm

Famose, J.-P. (1990). Apprentissage moteur et difficulté de la tâche. Paris: Insep. 


\section{DOSSIER}

Fougeyrollas, P., et Boukala, M. (2009). Entretien avec Patrick Fougeyrollas. La nouvelle revue de l'adaptation et de la scolarisation, 45, 165-174.

Frank, M. (2006). Le cas de Martin, atteint de cécité. Revue EPS, 319, 31-35.

Gagnon, Y. (2012). L'étude de cas comme méthode de recherche. Québec: Presses de l'université du Québec.

Gardou, C. (2005). Fragments sur le handicap et la vulnérabilité. Toulouse: Érès.

Garel, J.-P., Siros M., et Gomez F. (1992). Enseigner les sports collectifs aux aveugles. Suresnes: Éditions du Cnefei.

Garel, J.-P., et Siros, M. (1992). S'informer pour agir. La prise d'information des joueurs aveugles dans la pratique du handball et du basketball. Le Courrier de Suresnes, 56, 61-67.

Garel, J.-P. (dir.). (1996). Éducation physique et handicap moteur. Paris: Nathan.

Garel, J.-P. (2001). Des collégiens comme les autres. L'intégration en éducation physique et sportive d'élèves présentant un handicap mental. DVD, 40 minutes. Suresnes: INSHEA.

Garel, J.-P. (2003). Des jeux sans voir. Revue EPS1, 115, 19-20.

Germez, D. (2001). Agir dans le monde - Les jeux collectifs: une aide à l'intégration des enfants aveugles. Mémoire pour le diplôme du Capsais. Suresnes: INSHEA.

Gomez, F. (1987). Sports collectifs avec des adolescents aveugles. L'éducation physique et sportive des enfants et adolescents déficients visuels et aveugles. Actes des journées d'étude, 17-18-19 novembre 1986. Le courrier de Suresnes, 40-50.

Gomez, F., et Siros, M. (1996). L'enseignement des sports collectifs aux aveugles. Handicapés physiques et inaptes partiels en EPS. Dossier EPS, 23, 159-163. Paris: Éditions Revue EP\&S.

Gouat, J.-P. (1999). Comment les activités physiques et sportives peuvent-elles être un vecteur d'intégration pour des enfants d'établissements spécialisés handicapés mentaux. Mémoire pour le diplôme de DDEEAS. Suresnes: INSHEA.

Guillerme, J. (2008). L'art du projet: histoire, technique, architecture. Paris: Mardaga.

Huberman, A. M. (1973). Comment s'opèrent les changements en éducation: contribution à l'étude de l'innovation. Paris: Unesco.

Lapeyronnie, D. (2014). Pour une École innovante. Synthèse des travaux du Conseil national de l'innovation pour la réussite éducative. Rapport à madame la ministre de l'Éducation nationale, de l'Enseignement supérieur et de la Recherche. http://www.ladocumentationfrancaise.fr/var/storage/rapports-publics/144000674.pdf

Lucidarme, T. (2013). Valoriser et développer l'innovation. De l'innovation incrémentale à l'innovation visionnaire de rupture. Paris: Vuibert.

Marcellini, A. (2005a). Pour le développement des pratiques sportives mixtes: une application du principe de discrimination positive? EPS, sport et handicap, quelles activités adaptées, à quelles conditions? (pp. 6-9). Actes du forum international de l'éducation physique et du sport - 4-5-6 novembre 2005 - Cité internationale. Paris. http://Www.epsetsociete.fr/EPS-sport-et-handicap-quelles

Marcellini, A. (2005b). Des vies en fauteuil... Usages du sport dans les processus de déstigmatisation et d'intégration sociale. Paris: CTNERHI.

Parlebas, P. (1981). Contribution à un lexique commenté en science de l'action motrice. Paris: Insep. 
Pastré, P. (1999). Travail et compétences: un point de vue de didacticien. Formation emploi, 67, 109, 125.

Pastré, P., Mayen, P., et Vergnaud, G. (2006). La didactique professionnelle. Revue française de pédagogie, 154, 145-198. http://rfp.revues.org/157

Prud'homme, M.-H., et Goupil, G. (2001). Un moyen de favoriser l'insertion sociale: le groupe de jeu intégré. Handicap - revue de sciences humaines et sociales. Paris: CTNERHI.

Sarloute, M. (2015). Sport et handicap physique au féminin. Contrepied. [Hors série 12], 44.

Schumpeter, J. (1999). Théorie de l'évolution économique. Paris: Dalloz. (CEuvre originale publiée en 1912).

Séguillon, D. (2015). La surdité, entre handicap et culture. Contrepied [Hors série 12], 32-33.

Taylor, J.B. (1970). Introducing social innovation. Journal of Applied Behavioral Science, 1(6), 69-77.

Valet, A. (2013). Sport, inclusion et innovation: le cas italien du baskin (2001-2013). Thèse. Université Claude Bernard - Lyon I. <https://tel.archives-ouvertes.fr/ tel-01069780/document>

Valet, A. (2015). Le sport au défi de la logique inclusive. L'exemple du baskin. Contrepied [Hors série 12], 28-29. <http://www.epsetsociete.fr/Le-sport-au-defi-de-la-logique>

Von Bülow, N. (2012). L'innovation en France: un système en échec. Paris: Terra Nova. http://tnova.fr/notes/l-innovation-en-france-un-systeme-en-echec

Yin, R. (1994). Case of study research: design and methods. London: Sage. 